\title{
Parasitismo de Amblyomma fuscum (Acari: Ixodidae) em humanos
}

\author{
Parasitism of Amblyomma fuscum (Acari: Ixodidae) on humans \\ Sandro Marques ${ }^{1}$ Rogério Dal Col $^{1}$ Marco Otávio de Matos Júnior ${ }^{1}$ \\ Elisabeth Fernandes Bertoletti Gonçalves ${ }^{1}$ Adriano Pinter ${ }^{2}$ \\ Marcelo Bahia Labruna ${ }^{3}$
}

\begin{abstract}
- NOTA -
RESUMO

Amblyomma fuscum é uma espécie rara de carrapato, recentemente revalidada, com poucos registros de ocorrência na literatura. Neste trabalho, são informados dois registros de parasitismo de A. fuscum em seres humanos: um no Estado de São Paulo (SP) e outro possivelmente oriundo do Estado de Santa Catarina (SC), Brasil. Tratam-se dos primeiros registros de parasitismo em humano por esta espécie de carrapato. Um espécime adulto fêmea de A. fuscum foi coletado quando encontrava-se fixado à palma da mão de um dos autores deste trabalho, no município de Guarujá, SP. O segundo espécime, também adulto fêmea, foi coletado quando se encontrava fixado ao tornozelo de uma cidadã da cidade de São Paulo, que havia retornado de Lagoa da Conceição, em Florianópolis, SC, três dias antes. O presente registro de $A$. fuscum no município de Guarujá, SP, e outro possivelmente

reports two records of human parasitism by A. fuscum, one in the state of São Paulo (SP), and the other possibly from the state of Santa Catarina (SC), Brazil. These are the first reports of $\boldsymbol{A}$. fuscum on humans. An adult female specimen of $\boldsymbol{A}$. fuscum was collected while attached to the palmar area of the hand of one of the authors of the present study, in Guarujá, SP. The second specimen, also an adult female, was collected while attached to the ankle of a resident of São Paulo city, who had returned three days before from Lagoa da Conceição, Florianópolis Municipality, SC. The present report of A. fuscum in Guarujá, SP and another one, possibly from Florianópolis, $S C$, reinforce previous reports of this tick species in these two Brazilian states. Although the adult stage of A. fuscum has been primarily associated with reptiles, other reports on mammals (Carnivora and Edentata) and the present report on humans suggest low host specificity.
\end{abstract} oriundo de Florianópolis, SC, reforça os achados prévios deste carrapato nesses dois Estados, especialmente em região costeira. Embora o estágio adulto de A. fuscum tenha sido associado primariamente com répteis, os vários registros em mamíferos (Carnivora e Edentata) e o presente registro em seres humanos sugerem uma baixa especificidade parasitária.

Palavras-chave: Amblyomma fuscum, carrapato, parasitismo humano, São Paulo.

\section{ABSTRACT}

Amblyomma fuscum is a rare, recently revalidated tick species, with few records in the literature. The present study
Key words: Amblyomma fuscum, tick, human parasitism, São Paulo.

O carrapato Amblyomma fuscum Neumann, 1907 foi descrito a partir de um macho coletado em Boa constrictor, na América do Sul, sem especificações da localidade. A fêmea foi descrita por ARAGÃO (1936), através de espécimes coletados em um lagarto, no Sul do Brasil. Redescrita recentemente por BARROS-

${ }^{1}$ Laboratório de Identificação e Pesquisa de Fauna Sinantrópica da Prefeitura Municipal de São Paulo, São Paulo, SP, Brasil.

${ }^{2}$ Programa de Pós-graduação em Epidemiologia Experimental e Aplicada a Zoonoses, Universidade de São Paulo (USP), São Paulo, SP, Brasil.

${ }^{3}$ Departamento de Medicina Veterinária Preventiva e Saúde Animal, Faculdade de Medicina Veterinária e Zootecnia (FMVZ), USP, Av. Prof. Orlando Marques de Paiva, 87, 05508-000, São Paulo, SP, Brasil. E-mail: labruna@usp.br. Autor para Correspondência. 
BATTESTI et al. (2005), A. fuscum possui poucos registros de ocorrência e hospedeiros. Segundo esses autores, os únicos registros com localidade definida para A. fuscum remetem aos municípios de Santa Vitória do Palmar, Passo da Areia, Rio Grande, Rio Telha e Triunfo, no Estado do Rio Grande do Sul, e Cananéia, Iguape, Praia Grande e Rosana, no Estado de São Paulo. Há ainda um registro para o Estado de Santa Catarina (município não definido), um para Recife, no Estado de Pernambuco (CUNHA et al., 1999), e um mais recente para Pelotas, no Rio Grande do Sul (BRUM et al., 2003).

Répteis têm sido descritos como hospedeiros preferenciais para o estádio adulto de $\boldsymbol{A}$. fuscum (GUIMARÃES et al., 2001; GUGLIEMONE et al., 2003; BARROS-BATTESTI et al., 2005), com registros para as espécies Boa constrictor, Drymarchon corais (= Coluber corais), Clelia clelia, Tupinambis teguxim e para um lagarto (Squamata) de espécie não identificada. Outros registros para adultos de A. fuscum foram no anfíbio Bufo arenalis (Anura) (SINKOC \& BRUM, 1997) e nos mamíferos Cerdocyon thous, Canis familiaris (Carnivora) e Dasypus septemcinctus (Edentata) (BRUM et al., 2003; BARROSBATTESTI et al., 2005). O único registro para estágio imaturo de A. fuscum é de uma ninfa em Didelphis aurita (Didelphimorphia) (BARROS-BATTESTI et al., 2005).

Neste trabalho, são relatados os primeiros registros de parasitismo humano por A. fuscum. Em 07 de fevereiro de 2005, em torno das 09h 30min, um dos autores deste trabalho (R.D.C.) coletou um espécime fêmea de carrapato que estava fixado na palma de sua mão. A constatação do carrapato ocorreu quando o autor caminhava pela trilha da Praia Branca, município de Guarujá, Estado de São Paulo (SP). O local tem características de região litorânea e de manguezal e, apesar da vegetação nativa de Mata Atlântica existente, nenhum animal foi visualizado no momento da constatação do carrapato ou durante o percurso pela trilha. No momento da coleta, a temperatura estava em torno de $30^{\circ} \mathrm{C}$, com céu aberto e alta incidência de raios solares.

Em 08 de janeiro de 2006, uma cidadã, moradora da área urbana da cidade de São Paulo, coletou um espécime de carrapato que estava fixado em seu tornozelo direito. A constatação do carrapato ocorreu quando a cidadã estava fazendo uma limpeza do interior de seu veículo. A pessoa havia retornado a São Paulo, dirigindo este mesmo veículo, três dias antes (05 janeiro 2006). O local de procedência era o Centro dos Araçás, na Lagoa da Conceição, em Florianópolis, Estado de Santa Catarina (SC). Segundo depoimento da própria cidadã, o veículo ficou fechado e intocável na garagem de sua residência entre os dias 5 e 8 de janeiro de 2006. Como ela nunca havia constatado a presença de carrapatos em sua residência ou em seu veículo anteriormente, ela sugeriu que este carrapato seria procedente do local visitado em Santa Catarina, por se tratar de uma área de preservação natural.

Os dois espécimes coletados foram fixados em álcool 70\%, sendo ambos identificados como fêmea de $\boldsymbol{A}$. fuscum, segundo BARROS-BATTESTI et al. (2005). Dorsalmente, apresenta um escudo de coloração marrom amarelada, com bordas acobreadas e as porções laterais e posterior contêm ornamentação de coloração leitosa. Este padrão de ornamentação é melhor visualizado quando se umedece o escudo com água ou álcool. As pontuações são poucas, profundas e concentradas nas porções laterais (Figura 1a). Ventralmente, há dois espinhos curtos e largos na coxa I, e um espinho curto e arredondado em cada uma das coxas II-IV (Figura 1b). Os dois espécimes foram depositados no Acervo de Fauna Sinantrópica do Laboratório de Identificação e Pesquisa de Fauna Sinantrópica da Prefeitura Municipal de São Paulo (Tombos 234 e 1392).

O presente registro de $\boldsymbol{A}$. fuscum no município de Guarujá, SP, e o outro possivelmente oriundo de Florianópolis, SC, reforçam os achados prévios deste carrapato nesses dois Estados, especialmente em região costeira. Embora o estádio adulto de $\boldsymbol{A}$. fuscum tenha sido associado primariamente com répteis (GUIMARÃES et al., 2001; GUGLIEMONE et al., 2003; BARROS-BATTESTI et al., 2005), os vários registros em mamíferos (Carnivora e Edentata) e o presente registro em um dois seres humanos sugerem que este carrapato tenha uma baixa especificidade parasitária.

\section{AGRADECIMENTOS}

Ao Conselho Nacional de Desenvolvimento Científico e Tecnológico (CNPq), pela Bolsa de Produtividade de Pesquisa do autor M. B. Labruna. 


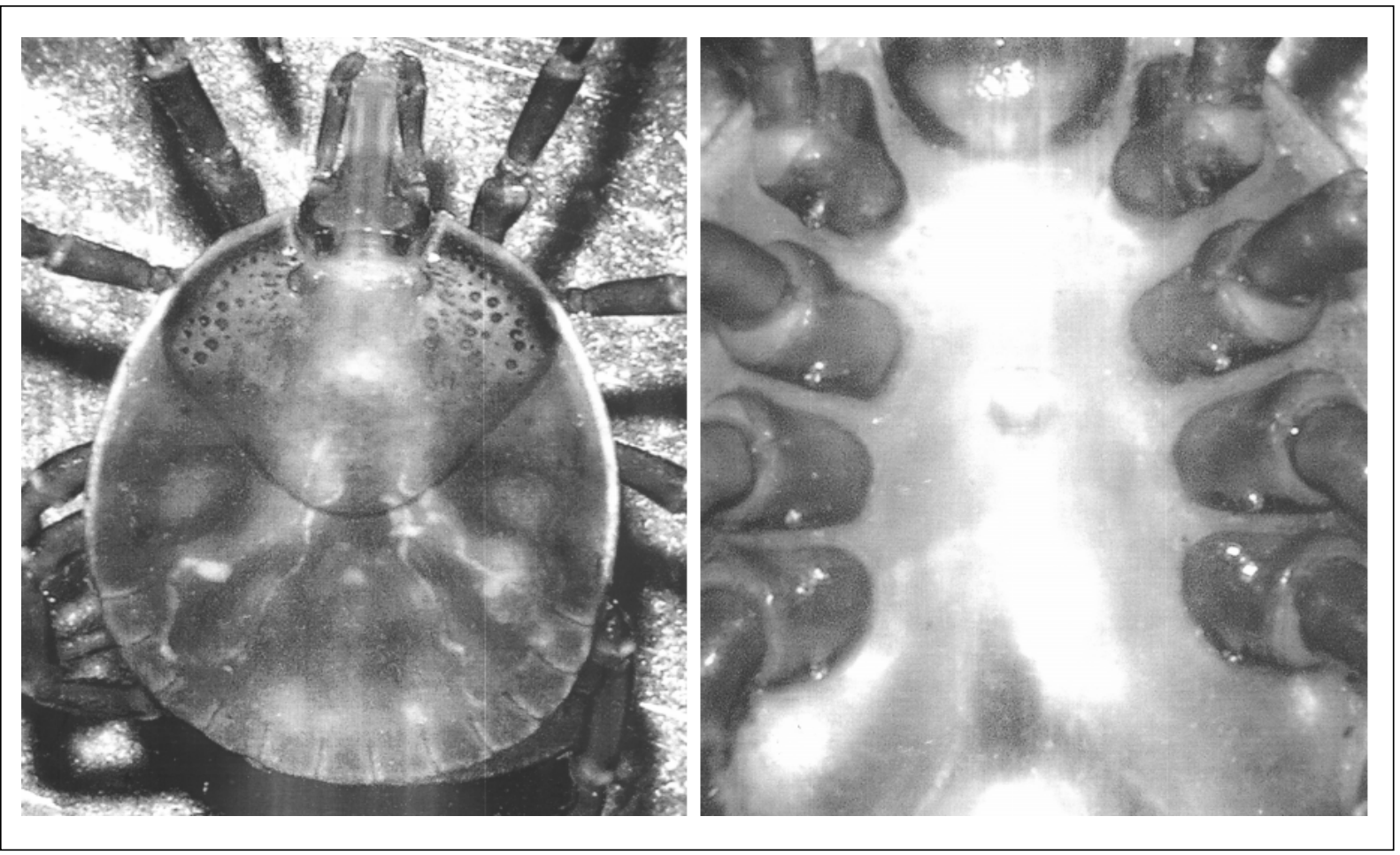

Figura 1 - Fêmea adulta de Amblyomma fuscum: Escudo (A) e coxas I-IV (B).

\section{REFERÊNCIAS}

ARAGÃO, H.B. Ixodidas brasileiros e de alguns países limítrofes. Memórias do Instituto Oswaldo Cruz, v.31, p.759-843, 1936.

BARROS-BATTESTI, D.M. et al. Redescription of Amblyomma fuscum Neumann, 1907 (Acari: Ixodidae), a rare South América tick confirmed in Brazil. Systematic Parasitology, v.61, p.85-92, 2005.

BRUM, J.G.W. et al. Ixodidae de mamíferos silvestres atendidos no Núcleo de Reabilitação da Fauna Silvestre, UFPEL. Arquivos do Instituto Biológico, v.70, n.2, p.211-212, 2003.

CUNHA, M.C.A.L. et al. Ocorrência de Amblyomma fuscum Neumann, 1907 (Acari: Ixodidae) em Boa constrictor Linnaeus,
1758 (Reptilia: Boidae) no estado de Pernambuco, Brasil. Entomologia y Vectores, v.6, p.577-579, 1999.

GUGLIELMONE, A.A. et al. Ticks (Acari: Ixodidae) of the Neotropical Zoogeographic Region. Houten, Atalanta: International Consortium of Ticks and Tick-borne Diseases, 2003. 173p.

GUIMARÃES, J.H. et al. Ectoparasitos de importância veterinária. São Paulo: Plêiade/FAPESP, 2001. 218p.

SINKOC, A.L.; BRUM, J.G.W. Ocorrência de Amblyomma fuscum Neumann, 1899 e Amblyomma humerale Koch, 1844 (Acari: Ixodidae) em Bufo arenalis no estado de São Paulo, Brasil. Ciência Rural, Santa Maria, v.27, p.339-340, 1997. 